\section{Shun Xu: a benevolent doctor}

Submitted Apr 17, 2018. Accepted for publication Apr 23, 2018.

doi: $10.21037 /$ jtd.2018.04.164

View this article at: http://dx.doi.org/10.21037/jtd.2018.04.164

Upon entering Professor Shun Xu's office, the first in sight is an exquisitely framed calligraphy scroll on the wall, which reads "benevolence, medical skills, medical ethics, and patients are of paramount importance". Prof. Xu (Figure 1) is the director of the Department of Thoracic Surgery at the First Hospital of China Medical University (FHCMU). He asked a friend with expertise in calligraphy to write this principle that he has upheld for many years and used to exhort young doctors (Figure 2).

Prof. Xu has helped out numerous patients with his extraordinary skills and scalpels. For him, however, medical science is not simply about skills; rather, a real doctor should also be able to relieve patients from sufferings with benevolence and humanitarian care. What he's been doing over the past three decades exemplifies the spirit conveyed by the 16 Chinese characters on the scroll.

\section{Expert's introduction}

Shun $\mathrm{Xu}$, professor, doctorate supervisor, director of the Department of Thoracic Surgery of the First Affiliated Hospital of China Medical University, and director of Lung Cancer Research Department of Cancer Institute of China Medical University. He also serves as the chairman of Liaoning Society of Thoracic Surgery, vice chairman of the International Conference on Thoracic Surgery 2017 \& the Fifth National Cancer Center Annual Meeting, member of the Chinese Society of Thoracic and Cardiovascular Surgery, member of the Lung Cancer Research Group of the Chinese Society of Thoracic and Cardiovascular Surgery, member of the Mediastinal and Thoracic Surgical Experts Committee of the Chinese Association of Thoracic Surgeons, vice chairman of Liaoning Association of Surgeons, vice chairman of Liaoning Provincial Anticancer Association, chairman of Shenyang Society of Thoracic Surgery, standing committee member of the China Breast Cancer Branch of China International Exchange and Promotion Association for Medical and Healthcare, standing committee member of the Thoracic Surgery Branch of China International Exchange and Promotion

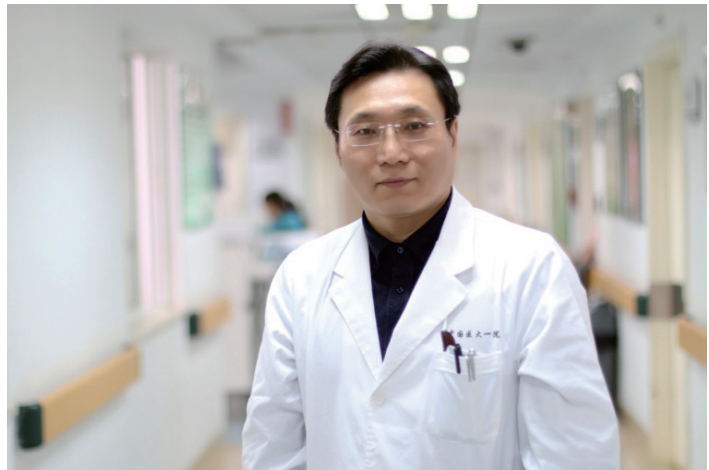

Figure 1 Prof. Shun Xu.

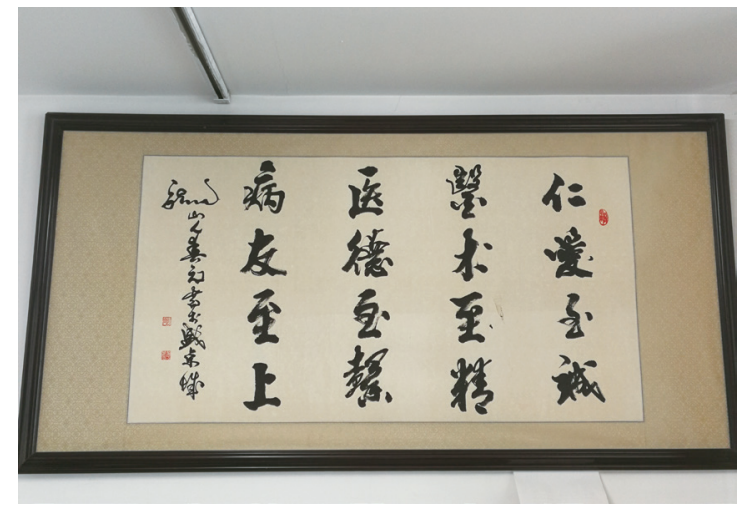

Figure 2 The calligraphy scroll on the wall of Prof. Shun Xu's office displays the principle upheld by him over the past years. The piece of writing is actually an acrostic. The first character of each line forms a sentence that means "Doctors treat patients with love", which is the best interpretation of medical science.

Association for Medical and Healthcare, deputy chief of the Thoracic Surgery Group of the ERAS Branch of China International Exchange and Promotion Association for Medical and Healthcare, president of Liaoning Provincial Lung Cancer Prevention and Treatment Association, and secretary-general of the Jieping Wu Medical FoundationHouwen Li Lung Cancer Education Development Foundation. He was awarded the honorable title of "Famous 
Doctor in Liaoning" by Liaoning Provincial Health and Family Planning Commission, Liaoning Provincial Medical Association, and Liaoning Provincial Medical Doctor Association in 2017.

He has long been committed to the basic and clinical research of thoracic tumors and the standardized and precise treatment of lung cancer, esophageal cancer, and mediastinal tumors. He is particularly skillful in the early diagnosis and minimally invasive treatment of lung cancer and the multi-disciplinary diagnosis and treatment and comprehensive treatment of advanced lung cancer. He has rich experiences in the diagnosis and treatment of complex and severe thoracic diseases. He is good at conventional open surgeries and 2D/3D thoracoscopic minimally invasive lobectomy, segmentectomy, lung cancer radical surgery, and minimally invasive treatment for esophageal benign and malignant tumors, mediastinal tumors, pneumothorax, and pulmonary bulla.

As an active promoter and practitioner of EARS in Liaoning Province and Northeastern region, his current work focuses in EARS, tubeless video-assisted thoracoscopic surgery (VATS) under anesthesia with spontaneous ventilation for chest tumors, and Da Vinci surgery.

$\mathrm{He}$ is the principal investigator or investigator of many research projects supported by ministries or Liaoning province. He has published many articles in SCI-indexed journals. As one of the corresponding authors, he published an article in the Lancet in 2017. He was the winner of one Liaoning Province Medical Science and Technology Progress Award (third grade) and the holder of an innovation patent. $\mathrm{He}$ is a contributor of Demonstrations of Early Diagnosis and Multidisciplinary Treatment of Lung Cancer (published by the People's Medical Publishing House), translator-inchief of Lung Cancer: A Multidisciplinary Approach to Diagnosis and Management, and deputy editor-in-chief of Technical Essentials of Thoracoscopic Surgery.

\section{Benevolence}

\section{"Kindness and virtue are the core of being a good doctor"}

Like many people, Prof. Xu chose to study medicine simply because he wanted to safeguard the health of his family members. When he was young, his baby sister was diagnosed with congenital heart disease. His parents took her to see famous doctors from place to place but to no avail, and his father's health deteriorated since then. The happy family was plagued by the diseases of his father and younger sister.
"As the eldest son, I decided to study medicine to safeguard the bealth of my family members at the time. At first, I wanted to become a cardiac surgeon, but inspired by my teacher professor Houwen Li, I followed his steps to study thoracic surgery, and has been a thoracic surgeon for 30 years" said Prof. Xu.

After graduating from the medical school, he immediately arranged a surgery for his younger sister, who was about 20 at the time. The surgery was very successful, and her own child has grown up now.

Later, he wanted to safeguard the health of more people, not only his family members. He never forgot the motto that "Kindness and virtue are the core of being a good doctor". "As a doctor, I am convinced that doctors must be kind-hearted, and this is by no means a cliche. Without benevolence, one can only regard doctor as a job rather than career. To become a respectable doctor, benevolence is indispensable," he said.

"Safety is a basic demand of patients. Therefore, it is important to be a safe doctor first. Surgeons need to use scalpels to treat diseases, which, however, is invasive. They must be able to keep surgical traumas within safe limits." Prof. Xu pointed out.

The application of new technologies should prioritize the safety of patients, and it is necessary to select the right patients. New technologies are a double-edged sword, and they can be good but also risky. Patient safety is the top priority, and risks should try to be minimized for the best interest of patients.

"Young doctors should not pursue individual heroism but choose the safest method for patients, and know their own limitations and select the most suitable professionals to take care of different patients." said Prof. Xu. Most importantly, it is better to ask for help when necessary than deal with problems alone. A good surgeon is not afraid of admitting "I don't know", "I didn't do that before", "Please help me", "Please give me some advice", as he/she puts the safety of patients above his/her self-esteem and reputation. Nothing is more important than life.

The progress of medical science has reduced but has not yet eliminated the pain of patients. Doctors need to minimize the pain and trauma by maximizing the curative effect, which is required by the era and the social development.

\section{Pursuit of perfection}

\section{"Be an artist who can save life and a prophet who can prevent disaster"}

On November 16, 2017, in the operating room of 
FHCMU, robotic resection of a mediastinal tumor was successfully performed by the team of Prof. Xu (Figures 3,4). This was for the first time the Da Vinci robotic surgical system was used in FHCMU. The operation was visualized and operated through three $1.0-1.5 \mathrm{~cm}$ ports on the right chest wall. The tumor was extracapsular dissected and then removed with minimal bleeding. The operation lasted for about one hour, and the patient became ambulatory four hours after the surgery. After the drainage tube was removed on the second postoperative day, the patient was discharged on the third day.

This was unbelievable in the past but now becomes true stories. No bloody and lengthy operation was needed, and the patient was not bedridden for a long period of time. The surgery was a perfect combination of minimally invasive surgery, enhanced recovery after surgery (ERAS), and tubeless anesthesia!

This was a milestone operation and marked the beginning of a new era of minimally invasive thoracic surgery in FHCMU. This new chapter is about to be written in the history of FHCMU and of Northeast China.

Medicine is vast and complicated, and scientific and technical explorations in this field never stop.

\section{Open the door to minimally invasive surgery}

The year of 2008 witnessed the 29th summer Olympic Games in Beijing and the celebration of the 100th anniversary of FHCMU; in the same year, the Second Department of Thoracic Surgery was established, and Prof. $\mathrm{Xu}$ served as its director. The new department focused on minimally invasive surgery, with an attempt to combine traditional surgeries with minimally invasive surgery. After the establishment of the department, Prof. $\mathrm{Xu}$ and his team have made a number of breakthroughs. As one of the pioneers of minimally invasive surgery in FHCMU, Prof. $\mathrm{Xu}$ was the surgeon who performed the first thoracoscopic lobectomy, Nuss-procedure for funnel chest, and mediastinoscopic surgery in the hospital. Today, over $90 \%$ of surgeries performed in the department are minimally invasive. The majority of patients can be successfully discharged in three or four days after surgery.

Minimally invasive surgery is a revolution in surgery. Thoracoscopic technology was introduced into China in 1992. As one of the promoters of minimally invasive surgery in Japan, Prof. Naruke from Japanese National Cancer Center closely cooperated with Prof. Houwen Li at

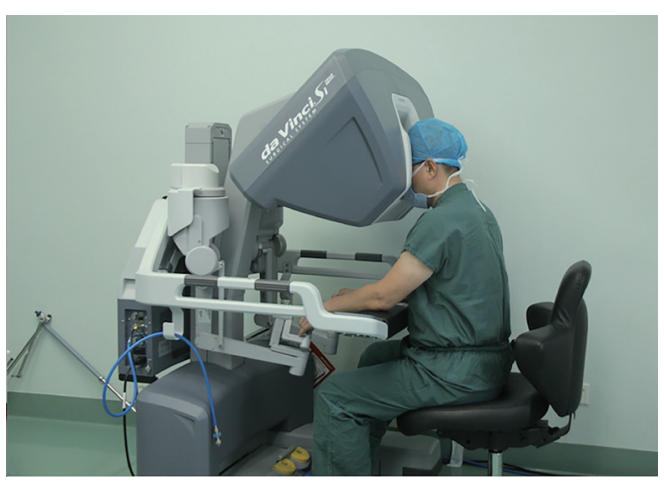

Figure 3 Prof. $\mathrm{Xu}$ is performing thymoma resection by using the Da Vinci robotic surgical system.

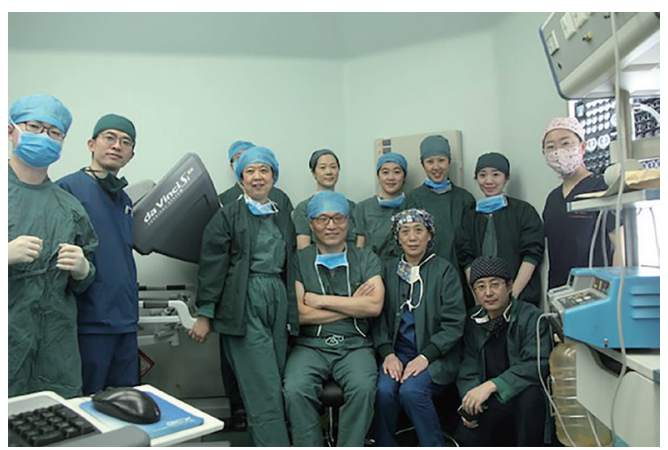

Figure 4 The team of the Da Vinci robotic surgery. The surgeon is Prof. $\mathrm{Xu}$, and the surgery is supported by Prof. Hong Ma's team from the Department of Anesthesiology and the nursing team led by Ms. Li Li.

FHCMU and performed thoracoscopic resection of lesions in a patient with metastatic lung tumor.

"For many people at that time, it was unbelievable to remove the lesions via such small incisions. Thanks to the efforts of many pioneers such as Dr. Fianxing He at the First Affiliated Hospital of Guangzhou Medical University, VATS techniques had been gradually developed in China and has become bighly popular since 2005.” Said Prof. Xu.

Prof. Xu has foreseen the development trend of thoracic surgery long time ago, "I'm grateful that we are in an era of development and innovation." He was admitted to the foreign language medical class at China Medical University in 1981 for six years' study rather than the normal five years, due to his high scores. He studied foreign language in the first year, and received teaching in foreign language in the remainder five years. He went a step further than others and developed a creative way of thinking. When he graduated 


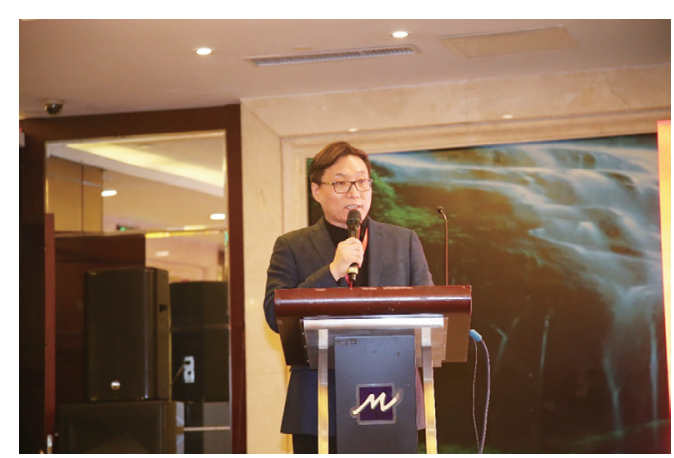

Figure 5 On December 1, 2017, Prof. Shun Xu' team, together with Prof. Jianxing He's team, sponsored the "Completely Minimally Invasive surgery for Recovery within 24 HoursTubeless \& ERAS Tour in China-Shenyang”.

from the university, China started to open to the world. $\mathrm{He}$ then passed examination to study abroad due to his language proficiency, and earned advanced thoughts and professional knowledge that broadened his horizon.

"After determining the direction, we just need to move ahead and blaze a trail despite of unpredictable bardships." There is no easy path to success. Prof. Xu has never balked at setbacks and challenges ahead. "I was confronted with doubts and difficulties when promoting minimally invasive surgery, but perseverance toward the right direction will eventually lead to success. There is light at the end of the tunnel."

Every stumble and failure helps us understand the life better, and all setbacks will eventually become shining medals.

\section{Implement ERAS}

"EARS is a promising idea and technology; as minimally invasive surgery has developed for 20 years. Our clinical practice has confirmed that it is feasible, at least in some selected patients." said Prof. Xu.

"The concept of ERAS runs through the entire bealth care process. The surgical team must make careful preparation before surgery, be skillful during surgery, and properly deal with all problems after surgery, with love and benevolence persisting throughout the whole process." EARS is an optimized clinical pathway that optimizes the preoperative, intraoperative, and postoperative processes. With core targets of "no stress, no pain, and no risk" pursued by surgeons, it adopts a series of evidence-based perioperative optimization measures to reduce the physical and mental traumas of surgical patients, decrease postoperative complications, shorten hospital stay, and thus promote rapid recovery after surgery.

Perioperative airway management is particularly important for the prevention and treatment of perioperative pulmonary complications; thus, it is a strong guarantee for ERAS among surgical patients, particularly in the department of thoracic surgery.

In a traditional thoracic surgery, the commonly used anesthesia method is endotracheal intubation, during which the healthy lung remains working while the diseased lung is stopped to ensure the safety of the operation. However, endotracheal intubation may cause problems such as tracheal mucosa damage, vocal cord injury, and misplacement into the esophagus, which can induce many complications such as pulmonary infection. "In the past we had no choice because surgery required anesthesia and anesthesia inevitably lead to these problems. Today we have a solution."

At the end of 2016, with an attempt to implement ERAS, thoracotomy and thoracoscopic lung surgery under tubeless anesthesia with spontaneous ventilation were successfully performed in FHCMU, thanks to the close cooperation among multiple departments, marking a breakthrough in the concepts of both surgery and anesthesia in the hospital.

"Tubeless anesthesia with spontaneous ventilation is performed under deep sedation or combined anesthesia. It allows surgical procedures under regional block, followed by postoperative analgesia. Since no muscle relaxant is used in anesthesia, patients can maintain spontaneous breatbing, and no endotracheal intubation is needed to control ventilation. The patients can quickly become ambulatory after surgery, thus acbieving quick recovery." said Prof. Xu (Figure 5).

However, the implementation of tubeless anesthesia can be risky. Due to the inability to control the pathophysiological changes caused by thoracotomy and the inability to perform separation of the two lungs, patients may suffer from hypoxemia, hypercapnia, hypotension, severe arrhythmia, and other problems during the surgery, especially for patients with underlying disorder of the vital organs before surgery. In addition, since the diseased lung ceases to work, it is likely to cause fatal consequences if the secretions, blood, or some other fluids flow into the healthy lung, just like the process of drowning.

Then, how to overcome these problems?

Based on the clinical experiences in other hospitals, Prof. $\mathrm{Xu}$ and his team gave top priority to patient safety in the introduction of new technologies. With the support of the Department of Anesthesiology, Prof. Xu 


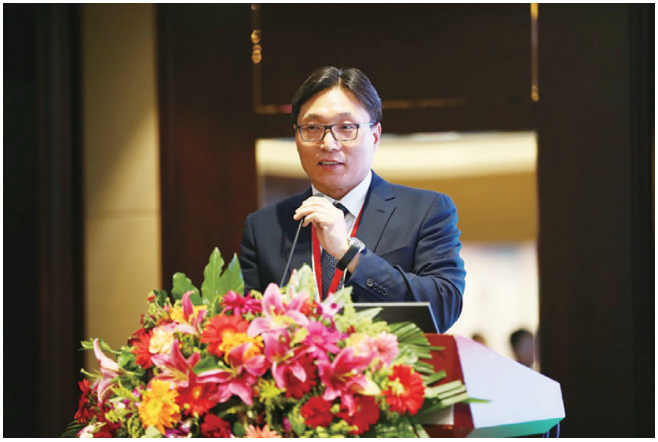

Figure 6 On September 15, 2017, Prof. Shun Xu chaired the Second Chinese-Japanese Summit Meeting on Oncology and gave a keynote lecture titled "Multidisciplinary Diagnosis and Treatment of Lung Cancer".

firstly made innovations in anesthesia mode. Secondly, Prof. $\mathrm{Xu}$ and his team made some fine management in the thoracic cavity to achieve truly precise anesthesia, including spraying anesthetics on the surface of lung, mediastinum, and diaphragm, local block of vagal nerve and intercostal nerves. The integration of precision anesthesia with minimally invasive surgery optimizes airway management and accelerates the development of ERAS.

"Doubts still exist: how can it work? Once the intraoperative bleeding cannot be controlled, patients may die on the operating table." However, today's anesthesia and minimally invasive techniques have reached high levels, which come from years of arduous practices.

"Doctors are making life-or-death decisions every day in hospitals. Although this is not limited to surgeons, many decisions of surgeons are made in minutes or even seconds. This requires an extraordinary ability of decision-making and also an art of seeing through death into life." Prof. Xu remarked. According to him, a good surgeon would take preventive measures and kill future disasters in the cradle.

\section{Lead the development of thoracic surgery}

"It is not difficult to be an ordinary doctor, you just need to follow conventions; to be an outstanding doctor, you must keep thinking; to be a competent leader, you must keep thinking and identify the right direction."

Life does not lack coincidences. November 16 of 2017 was a significant day for Prof. Xu. In the morning, he and his team completed the hospital's first Da Vinci robotassisted resection of mediastinal tumor. In the afternoon, he was elected chairman of the Liaoning Society of Thoracic Surgery.

"I'm lucky and honored to take over the post." Two weeks in office, Prof. Xu convened the first annual meeting of the 8th committee of the society, with a theme of "Minimally invasive surgery and enhanced recovery".

"Though it was windy and freezing outside, discussions in the meeting hall were enthusiastic." The meeting promoted the clinical application of ERAS in thoracic surgery under the multidisciplinary diagnosis and treatment mode and the application of video-assisted thoracic surgery under anesthesia with spontaneous breathing. Prof. Jianxing He, the developer of tubeless surgery, and Prof. Shun Xu and Prof. Hong Ma's team demonstrated the tubeless VATS radical treatment of lung cancer under anesthesia with spontaneous ventilation, respectively.

"Words are but wind, but seeing is believing. We can only convince others by our action, and then we can succeed in promoting the new technologies," said Prof. Xu.

"Tubeless thoracic surgery is a controversial technology that not everyone can master it. However, it will become a mega-trend over time. There are always pioneers, followers, doubters and even opponents during the emergence of new things. We should try to be the first two kinds of people. If we can't be pioneers, we can at least be followers to keep up with the time. The 19th CPC National Congress said we are entering the 'New Era'. Minimally invasive thoracic surgery and ERAS are also entering a new era."

Prof. Xu hoped to promote advanced theories and practices in the three provinces in northeastern China. "If we can't become national leaders, we can at least be leaders in the region and our hospital. The thoracic surgery department has succeeded, and other departments can do it as well."

Prof. Xu has maintained a good relationship with Japanese thoracic surgeons. Under his leadership, the "Chinese-Japanese Oncology Summit" has been held for two consecutive years for participants to exchange experiences in the diagnosis and treatment of lung cancer (Figures 6,7).

\section{Respect of medical ethics}

"That was a child at the age of my daughter. My reason told me to stay behind, but my conscience told me she was an angel awaiting help"

A 17-year-old girl who was battling with anterior mediastinal mass had been rejected by many hospitals, 


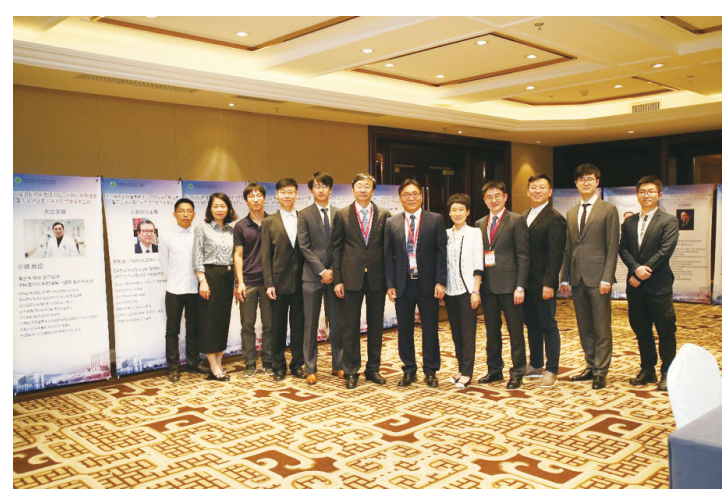

Figure 7 Prof. Shun Xu took a group photo with the meeting staff.

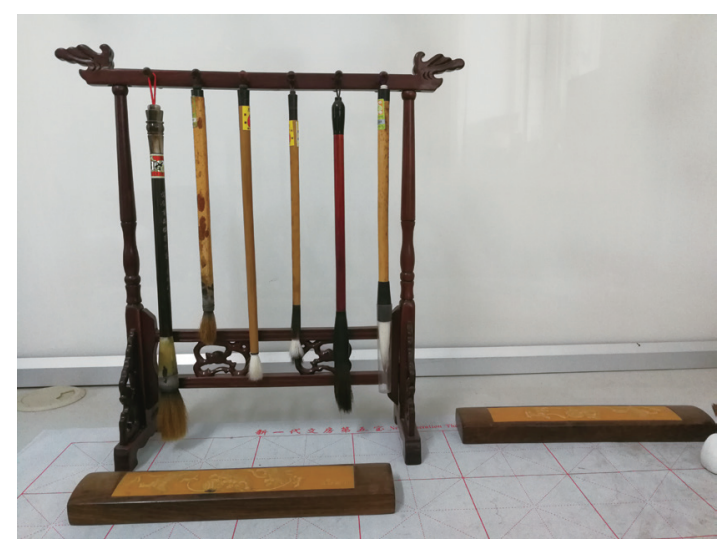

Figure 8 Prof. Xu loves reading and writing. Reading is thoughtprovoking and makes his mind profound.

and turned to Prof. Xu with the last shred of hope. The tumor had invaded the aorta, pericardium, and large vessels. When seeing the girl's lung CT images, he knew the surgery would be highly challenging and risky, and the patient was highly possible to die during the operation.

"As a surgeon, I was supposed to decline based on rational analysis, but when I turned to her, I met her crystal-like eyes full of anxious hope. She even tried to keep from blinking for fear of leaving a bad impression on the doctor." Prof. Xu faced internal struggles.

"That was a child at the age of my daughter. My reason told me to stay bebind, but my conscience told me she was an angel awaiting help. When I told her that I would give it a try, the excitement in her eyes was never forgettable."

Later, a nurse told Prof. Xu that the girl was calling different people after she was admitted to the hospital, telling them the same news "I am hospitalized and is going to receive a surgery. A doctor agreed to perform a surgery on me!"

The surgery was successful and the tumor was completely excised. But the patient eventually passed away due to heart failure after the surgery as her myocardial systolic function had been seriously affected by years of compression by the tumor. "Her death left me unsettled for a long time." said Prof. Xu.

There are two careers that are highly associated with virtue - teacher and doctor. Each medical staff must have a strong moral sense and strictly abide by medical ethics. A proverb goes as "doctors should care for patients like their parents", and Prof. Xu lives up to it.

\section{Patients first}

\section{"Patients' bealth and life are dependent on doctors"}

"We should treat a patient as a friend. When he is healthy, he may be able to make greater contributions to the society than us. When he is ill, we should help relieve him from physical and mental pains. Though doctors can't solve all medical problems, when the patients need our help, we should show willingness and sincerity to help them" said Prof. Xu.

"I often feel that China's medical education lacks bumanitarian care, but lays excessive emphasis on technology and curative effectiveness and even exaggerates the role of a specific treatment." He reckoned that life science is the most fascinating and mysterious science. It changes with time and environment and propels constant exploration. China's medical education is all about impersonal textbooks, and humanitarian care is rarely seen in clinical practices, during which communications between people actually are very important. Some people become good doctors, some are not and even assaulted by patients and their families.

"If we do not serve the country as a good official, then we become a good doctor to serve the people." For a man, what else is more important than life? Doctors maintain health, reduce pains, cure diseases, and prolong life. They are not ordinary but capable, insightful and motivated.

He often told other doctors that medical science is about face-to-face communications between people and is a career serving people. "You should win the trust of patients and give a sense of safety to them, because they put their life on your hands. For a doctor's growth, we offer sweat and tears while patients offer blood and life. Each and every experience and success is obtained from patients. Being aware of this, we can better sympathize with patients." (Figure 8). 


\section{Interview}

FTD: You have been committed to providing minimally invasive solutions in thoracic surgery. Can you introduce the advantages of minimally invasive surgery for ERAS compared to traditional open surgery?

Prof. Xu: The traditional open surgery requires a large incision to ensure a clear surgical field. The surgeon's hand or some auxiliary instruments must enter the patient's body via the incision. In minimally invasive surgery, the operative field is projected onto a television screen, and the auxiliary instruments are equivalent to the extension of the surgeon's hands. Thus, the surgery is performed by handeye coordination. When performing a traditional open surgery, the surgeon has to stare down at the surgical field throughout the operation; in contrast, when a surgeon is performing a minimally invasive surgery, his/her head is erect and his/her back is straight because he/she only needs to watch the screen.

Surgery is both a treatment process and a cause of iatrogenic trauma. After a surgery, it takes time for the recovery of both original trauma and surgical incision. Although minimally invasive surgery may cause some damage to the body, the incision on the body surface is significantly reduced. For example, the incisions of a minimally invasive surgery are about $3 \mathrm{~cm}$ in length, which are significantly shorter than those $(20-30 \mathrm{~cm})$ in an open surgery, enabling the patient to recover more quickly.

\section{FTD: What's the relationship between minimally invasive surgery and traditional surgery?}

Prof. Xu: Minimally invasive surgery has been developed on the basis of traditional surgery. It is very difficult for a surgeon without solid knowledge and good skill in traditional surgery to perform a thoracoscopic surgery directly. When any unexpected situation occurs, only surgeons with capabilities of traditional open surgery can manage it well. Thus, only technically competent and wellgrounded surgeons can seize the new opportunities in an era of "minimally invasive".

FTD: As one of the pioneers of minimally invasive surgery in the FHCMU, what difficulties did you experience in leading the team to perform minimally invasive surgery? How did you and your team solve them?

Prof. Xu: First of all, the medical staff needed to change their views on the surgery, which replaces naked eyes with camera and video and uses elongated surgical instruments instead of direct touch. Meanwhile, it was also necessary to change the principle of "large incision and complete exposure" emphasized in traditional surgical procedures. The new technology has allowed the clear visualization of the lesion and its surrounding structures on the screen, so that the operation can be performed safely, accurately and easily.

Another difficulty was the lack of minimally invasive surgical instruments and thoracoscopic devices. In the beginning, we had to perform thoracoscopic surgery in many cases by using instruments for conventional thoracotomy. Instead of complaining, we successfully moved up our initial learning curve based on the existing hardware conditions and our self-efficacy. Finally, we safely and efficiently performed the first minimally invasive surgery in our hospital, and today minimally invasive surgery has become a routine operation in our center.

\section{FTD: Why do you believe that ERAS will be widely accepted by patients?}

Prof. Xu: The advances in minimally invasive surgery have paved the way for ERAS, and minimally invasive surgery is a prerequisite for the development of ERAS.

Furthermore, ERAS is also an inevitable requirement in a new era. In the past, most of the patients would not visit a doctor until they became symptomatic; with the improvements in socioeconomic status, more diseases are identified during health check-ups and people can timely seek medical treatment before the onset of symptoms and signs. They are more demanding for the quality of life after the operation, and their desire for returning to society, life, and work is more urgent. They require smaller, more beautiful incisions that allow a similar or even better life experience. The patients' growing yearning for a better life after the operation is reinforcing the advances in minimally invasive surgery and ERAS.

FTD: What improvements can be brought about by ERAS? How bas ERAS developed in China?

Prof. Xu: For patients visiting our department, ERAS-based interventions during the perioperative period can improve surgical tolerance, reduce pain, lower the incidences of postoperative complications, promote the early recovery 
of respiratory and gastrointestinal functions, reduce hospitalization costs, improve patient satisfaction, and thus promote the rapid recovery of patients. The good outcomes after the clinical application of ERAS make this new idea more attractive. The concept of ERAS was introduced into China in 2007; since then, it has been widely used in many surgical settings. In 2015, the Chinese ERAS collaborative team was established, and the first national conference on ERAS was held in Nanjing. Thus, research and application of ERAS has entered a fast-track in China.

FTD: Airway management is an important part of ERAS. Compared with the Consensus on Airway Management in 2012, which focused on the "thoracic surgery", the 2016 edition has adopted a more "multidisciplinary" approach. What's your comment?

Prof. Xu: The importance of airway management has long been recognized by Chinese scholars. In 2012, many specialists in China developed the "Expert Consensus on Perioperative Airway Management in the Department of Thoracic Surgery". After several years of clinical practice, especially after the introduction of ERAS, we have gradually realized that airway management is not only the task of the department of thoracic surgery alone; rather, it requires the participation of multiple departments. Therefore, in 2016, a multidisciplinary team with members from the departments of anesthesiology, thoracic surgery, respiratory medicine, ICU, rehabilitation, and nursing was formed to revise the 2012 edition of "Expert Consensus on Perioperative Airway Management in the Department of Thoracic Surgery"; accordingly, the title of this document was changed to "Expert Consensus on A Multidisciplinary Approach to Perioperative Airway Management". The transition from "thoracic surgery" to "multidisciplinary" in the name of consensus reflects our deeper understanding of perioperative airway management and calls for further cooperation among multiple departments.

\section{FTD: Airway inflammation is a central link of the postoperative complications of thoracic surgery. It is also particularly challenging throughout the recovery of patients. What measures will be taken to manage airway inflammation?}

Prof. Xu: There are two types of inflammation: one is traumatic inflammation, caused by procedures such as tracheal intubation; the other is infectious inflammation, which may occur in patients with low immunity after surgical anesthesia or due to other reasons. Therefore, management of airway inflammation should both reduce trauma and prevent infections. The commonly used drugs include anti-infective agents and drugs for airway inflammation.

For example, for patients at high risk of postoperative airway infection, antibiotics should be used prophylactically before surgery. Also, glucocorticoids have important clinical values for stress regulation by alleviating postoperative traumatic stress and reducing postoperative lung complications; furthermore, they can protect throat mucosa.

\section{FTD: In recent years, the Da Vinci Surgical System has been increasingly used in clinical settings. How do you think about the future of $\mathrm{Da}$ Vinci surgery?}

Prof. Xu: The Da Vinci Surgical System is currently the most advanced minimally invasive surgical treatment platform. It further promotes the clinical practice of ERAS based on multidisciplinary diagnosis and treatment mode in the field of thoracic surgery, increases the arsenal of minimally invasive surgery for thoracic surgery, and paves the way for the wider adoption of the concept of ERAS.

However, the Da Vinci system itself has not brought revolutionary and profound changes to surgery. If surgery is compared to art, whether it is good or bad depends on the artist's ingenuity and craftsmanship. It is not important whether the tool is electric or manual and whether the artist sits or stands at work. Nevertheless, I believe in the future and believe in the wisdom and intelligence of mankind. Robotics are constantly evolving. In the near future, surgical robots will be able to overcome the current technical deficiencies such as the improper force feedback during the operation and the high cost of the system. Perhaps, Nano robots may be implanted through tiny incisions and autonomously remove lesions in human body, thus achieving truly precise, minimally invasive treatment. By that time, the doctor's hands can be truly liberated and the surgery is no longer a daunting process.

Link to this interviewing video: https://v.qq.com/x/page/ r0522as0fzp.html.

\section{Acknowledgements}

We thank Dr. Yanbin Sun and Dr. Changbo Sun from the Department of Thoracic Surgery of the First Hospital 
of China Medical University for their help during the interview. We also thank Yuan Yu from AME Publishing Company for her tireless support behind the scenes, and Xuefang Mai from AME Publishing Company for her video editing.

Cite this article as: Dong J. Shun Xu: a benevolent doctor. J Thorac Dis 2018;10(Suppl 11):S1313-S1321. doi: 10.21037/ jtd.2018.04.164

\section{Footnote}

Conflicts of Interest: The author has no conflicts of interest to declare.

(Science Editor: Jie Dong, JTD, jtd@amepc.org) 